\title{
Spin-glass-like transition in the majority-vote model with anticonformists
}

\author{
Andrzej Krawiecki ${ }^{\mathrm{a}}$ \\ Faculty of Physics, Warsaw University of Technology, Koszykowa 75, 00-662 Warsaw, Poland
}

Received 29 September 2017 / Received in final form 27 November 2017

Published online 7 March 2018

(C) The Author(s) 2018. This article is published with open access at Springerlink.com

\begin{abstract}
Majority-vote model on scale-free networks and random graphs is investigated in which a randomly chosen fraction $p$ of agents (called anticonformists) follows an antiferromagnetic update rule, i.e., they assume, with probability governed by a parameter $q(0<q<1 / 2)$, the opinion opposite to that of the majority of their neighbors, while the remaining $1-p$ fraction of agents (conformists) follows the usual ferromagnetic update rule assuming, with probability governed by the same parameter $q$, the opinion in accordance with that of the majority of their neighbors. For $p=1$ it is shown by Monte Carlo simulations and using the Binder cumulants method that for decreasing $q$ the model undergoes second-order phase transition from a disordered (paramagnetic) state to a spin-glass-like state, characterized by a non-zero value of the spin-glass order parameter measuring the overlap of agents' opinions in two replicas of the system, and simultaneously by the magnetization close to zero. In the case of the model on scale-free networks the critical value of the parameter $q$ weakly depends on the details of the degree distribution. As $p$ is decreased, the critical value of $q$ falls quickly to zero and only the disordered phase is observed. On the other hand, for $p$ close to zero for decreasing $q$ the usual ferromagnetic transition is observed.
\end{abstract}

\section{Introduction}

Critical phenomena in complex networks have been a subject of broad interest in statistical physics in the last two decades [1]. In particular, ferromagnetic (FM) and spin-glass (SG) phase transitions in the generic equilibrium Ising model on complex networks have been studied both by Monte Carlo (MC) simulations [2-6] and various theoretical methods, e.g., mean-field (MF) approximation [7], belief-propagation algorithm [8,9] and replica method [10-14]. Much less is known on the possible phase transitions in the nonequilibrium counterpart of the Ising model, the majority-vote (MV) model $[15,16]$ in which the probability of the opinion flip of each agent (represented by a two-state spin), controlled by the level of internal noise, depends only on the sign of the resultant opinion of her neighbors rather than on its exact value. This is mainly due to the lack of the Hamiltonian for the model and the resulting shortage of the analytic methods. In most cases the simplest variant of the MV model with conformists (agents following with higher probability the opinion of the majority of their neighbors) was investigated, both on regular lattices [15-18] and various complex networks [19-23], and it was shown by means of MC simulations [15-23] and MF approximation [17,23] that it exhibits continuous ordering transition as the level of internal noise is decreased, although there is no exact proof that the observed FM phase is stable. Only recently the MV model

\footnotetext{
${ }^{\mathrm{a}}$ e-mail: akraw@if.pw.edu.pl
}

with a fraction of agents being anticonformists (following with higher probability the opinion opposite to that of the majority of their neighbors) has been investigated on regular lattices using MC simulations and antiferromagnetic (AFM) transition has been found [24]. Other extensions of the MV model which also enrich its critical behavior comprise, e.g., inclusion of heterogeneous agents [25], agents with independence [26], agents with more than two opinions [27] and with inertia (which leads to the occurrence of a discontinuous FM transition) [28].

In this paper the MV model in which a randomly chosen fraction $p$ of agents are anticonformists while the remaining agents are conformists is investigated on different complex networks, including heterogeneous scale-free (SF) networks $[29,30]$ and random Erdös-Renyi (ER) graphs [30,31]. On such networks, due to lack of translational symmetry, one cannot expect the occurrence of the AFM phase in the MV model, characterized by long-range ordering with periodically alternating layers of agents with opposite orientations. Instead, it is shown by means of MC simulations that for $p \rightarrow 1$ with decreasing level of the internal noise the model on networks with finite second moment of the degree distribution exhibits transition from a disordered phase to a SG-like phase characterized by short-range AFM ordering and lack of long-range ordering [32-34]. Since the Hamiltonian for the MV model does not exist and thus the free energy cannot be minimized it is not possible to show that for a given realization of the network and of the distribution of (anti)conformists 
any "ground" state corresponds to the SG-like phase. Nevertheless, the states (opinion configurations) of the model reached by slowly decreasing the level of internal noise exhibit high overlap in the SG-like phase for different initial conditions in the disordered phase and for different realizations of the internal noise which results in non-zero value of the $\mathrm{SG}$ order parameter. In contrast, for small $p$ the usual FM transition is observed in the model as the level of internal noise is decreased. Hence, the presence of anticonformists leads to higher diversity of the possible critical behavior of the MV model on complex networks.

\section{The model and methods of analysis}

\subsection{Majority-vote model with anticonformists on complex networks}

The MV model [15-23] is a stochastic model for the opinion formation in which agents represented by two-state spins $s_{i}= \pm 1, i=1,2, \ldots, N$ located in the nodes of a (possibly complex) network update their opinions (orientations) at discrete time steps with probability dependent on the opinion of the majority of their neighbors. In most cases the agents are conformists obeying the FM update rule in which the probability of the opinion flip per unit time (rate) is

$$
w_{i}\left(s_{i}\right)=\frac{1}{2}\left[1-(1-2 q) s_{i} \operatorname{sign}_{i}\right]
$$

where $0<q<1 / 2$ is the model parameter which controls the level of internal noise,

$$
\operatorname{sign}_{i}=\operatorname{sign}\left(\sum_{j \in n n_{i}} s_{j}\right), \operatorname{sign}(x)=\left\{\begin{array}{ccc}
-1 & \text { for } & x<0 \\
0 & \text { for } & x=0 \\
+1 & \text { for } & x>0
\end{array}\right.
$$

and $n n_{i}$ denotes a set of the nearest neighbors of the node $i$, i.e., of the nodes which are directly connected by edges with the node $i$. According to this update rule the agents assume the opinion of the majority of their neighbors with probability $1-q$ and the opposite opinion with probability $q$. Hence, the parameter $q$ is a measure of the agents' uncertainity in decision making, sometimes associated with an effective "social temperature" $\Theta$ via the relationship $1-2 q=\tanh (1 / \Theta)[17]$, which should not be misinterpreted as temperature characterizing equilibrium models. Instead, the MV model with the FM update rule (1) is a nonequilibrium model which can be regarded as an open Ising system in contact with two heat reservoirs, one with infinite temperature and the other one with zero temperature, with probability $2 q$ and $1-2 q$, respectively; besides, it does not obey detailed balance condition [15]. Nevertheless, MC simulations [15-23] reveal that it shows FM transition as the level of internal noise is decreased, and the critical value of the parameter $q$ can be evaluated in the MF approximation as [23]

$$
q_{c}=\frac{1}{2}\left(1-\frac{\sqrt{2 \pi}}{2} \frac{\langle k\rangle}{\left\langle k^{3 / 2}\right\rangle}\right)
$$

where $\langle k\rangle$ and $\left\langle k^{3 / 2}\right\rangle$ denote the mean degree and the moment of order $3 / 2$ of the degree distribution $p(k)$ of the network on which the model is defined.

In this paper an extension of the above-mentioned model is considered in which a randomly chosen fraction $p, 0 \leq p \leq 1$, of agents are anticonformists obeying the AFM update rule

$$
w_{i}\left(s_{i}\right)=\frac{1}{2}\left[1+(1-2 q) s_{i} \operatorname{sign}_{i}\right]
$$

i.e., for $0<q<1 / 2$ following with probability $1-q$ opinion opposite to that of the majority of their neighbors, and the remaining fraction $1-p$ of agents are, as usually, conformists obeying the FM update rule (1) and following with probability $1-q$ the opinion of the majority of their neighbors. It should be mentioned that in this model interactions between agents need not be symmetric: if two neighboring agents $i, j$ are conformist and anticonformist, respectively, agent $i$ tends to follow the opinion of the majority of her neighbors, including agent $j$, while agent $j$ tends to follow the opinion opposite to that of her neighbors, including agent $i$. It is known that in the MV model on regular lattices with both conformists and anticonformists AFM transition can occur [24]. In this paper the model is investigated on heterogeneous SF networks $[29,30]$ characterized by the degree distribution $p(k) \propto k^{-\gamma}, \gamma>2$, for $k \geq m$ and $p(k)=0$ for $k<m$ as well as on random ER graphs [30,31]. Such model shows some resemblance to the Ising model on complex networks with FM or AFM exchange integrals randomly, with certain probability, assigned to the edges, with an important difference that in the latter case interactions between neighboring spins are symmetric. The latter model is a generic equilibrium model for dilute spin glasses [11-14]. Thus, the main goal of this paper is to verify if, apart from the usual FM transition, the SG-like transition can occur in the nonequilibrium MV model under study.

\subsection{Methods of Monte Carlo simulation and analysis of results}

In order to verify the occurrence of the FM or SGlike phase transition MC simulations of the MV model under study on networks with the number of nodes $10^{3} \leq N \leq 10^{4}$ are performed using simulated annealing algorithm with random sequential updating of the agents' opinions. For each realization of the network and of the distribution of (anti)conformists simulation is started in the disordered phase at high $q$ with random initial conditions. Then the level of internal noise is decreased in small steps toward zero, and at each intermediate value of $q$, after a sufficiently long transient, the order parameters for the FM and SG-like transitions are calculated as averages over the time series of the opinion configurations. The results are then averaged over 100-500 (depending on $N$ ) realizations of the network and of the distribution of (anti)conformists.

The possible FM and SG-like transitions in the MV model under study are investigated in the same way as in the Ising model. The order parameter for the FM 
transition is the absolute value of the magnetization

$$
M=\left|\left[\left\langle\frac{1}{N} \sum_{i=1}^{N} s_{i}\right\rangle_{t}\right]_{a v}\right| \equiv\left|\left[\langle\tilde{M}\rangle_{t}\right]_{a v}\right|,
$$

where $\langle\cdot\rangle_{t}$ denotes the time average for the system on a given realization of a network and with a given realization of the distribution of (anti)conformists, and $[\cdot]_{a v}$ denotes average over different realizations of the network and of the distribution of (anti)conformists. The order parameter for the SG-like transition (henceforth called the SG order parameter) is the absolute value of the overlap parameter [32-34]

$$
Q=\left|\left[\left\langle\frac{1}{N} \sum_{i=1}^{N} s_{i}^{\alpha} s_{i}^{\beta}\right\rangle_{t}\right]_{a v}\right| \equiv\left|\left[\langle\tilde{Q}\rangle_{t}\right]_{a v}\right|
$$

where $\alpha, \beta$ denote two copies (replicas) of the system simulated independently with different random initial conditions. In the disordered phase both $M$ and $Q$ are close to zero. In the case of the FM transition both $M$ and $Q$ increase as $q$ is decreased. In the case of the SG-like transition the SG order parameter $Q$ increases as $q$ is decreased while the magnetization $M$ remains close to zero.

The critical value $q_{c}$ of the parameter $q$ for the FM and SG transitions can be determined from the intersection point of the respective Binder cumulants $U^{(M)}$ vs. $q$ and $U^{(Q)}$ vs. $q$ for systems with different numbers of agents $N$ [35], where

$$
\begin{aligned}
& U^{(M)}=\frac{1}{2}\left[3-\frac{\left\langle\tilde{M}^{4}\right\rangle_{t}}{\left\langle\tilde{M}^{2}\right\rangle_{t}^{2}}\right]_{a v}, \\
& U^{(Q)}=\frac{1}{2}\left[3-\frac{\left\langle\tilde{Q}^{4}\right\rangle_{t}}{\left\langle\tilde{Q}^{2}\right\rangle_{t}^{2}}\right]_{a v} .
\end{aligned}
$$

Besides, in the case of the second-order transition the respective cumulants for all $N$ should decrease monotonically with $q$, while in the case of the first-order transition they should exhibit minima as functions of $q$.

The ordering of agents' opinions can be investigated using the correlation function

$$
C(r)=\left[\left\langle s_{i} s_{j}\right\rangle_{\{(i, j): \operatorname{dist}(i, j)=r\}}\right]_{a v},
$$

where $\operatorname{dist}(i, j)$ denotes the length of the shortest path between the nodes $i, j$ and the average $\langle\cdot\rangle_{\{(i, j): \operatorname{dist}(i, j)=r\}}$ is over all pairs of nodes in a given realization of the network connected by a shortest path of length $r$. In the SG-like phase fast decay of its absolute value $|C(r)| \propto$ $\exp \left(-r / r_{0}\right)$ shows lack of long-range ordering, while oscillations of the curve $C$ vs. $r$ around zero indicate the presence of short-range AFM ordering [32-34].

\subsection{Properties of the spin-glass-like transition}

In the Ising model the SG transition occurs since, as the temperature is decreased, the system evolves toward a state with short-range ordering corresponding to a minimum of the energetic landscape [32-34]. Depending on the SG model the ground state can be degenerate or almost degenerate, and the energetic landscape can be characterized by many local minima corresponding to metastable states in which, particularly at low temperatures, the system can be trapped for exponentially long times. Nevertheless, due to averaging over different realizations of the network, distributions of the exchange integrals, initial spin configurations and realizations of thermal noise in equation (6) the SG order parameter increases with decreasing temperature. In order to speed up the equilibration process and, possibly, increase $Q$ improved algorithms of MC simulations can be used, e.g., parallel tempering [36-38] in which simulations are performed simultaneously at many different temperatures and from time to time the spin configurations are exchanged in such a way that the algorithm obeys detailed balance condition.

In contrast, the MV model is defined only via the local update rules (1) and (4) and there is no global "target function" (such as the Hamiltonian in the case of the Ising model) which should be minimized during MC simulation. In particular, there is not any "ground" state; hence, in principle, even for a fixed realization of the network and of the distribution of (anti)conformists the low- $q$ states reached using the simulated annealing algorithm with different initial conditions and realizations of the internal noise need not overlap. Moreover, there is no way to increase this overlap by applying systematically any version of the parallel tempering algorithm. Nevertheless, it turns out that in the MV model under study on networks with finite $\left\langle k^{2}\right\rangle$ for a narrow range of $p \rightarrow 1$ such overlap significantly increases with the decrease of $q$ and that the correlation function $C(r)$ in the low- $q$ states shows oscillations typical of the short-range AFM ordering, while the long-range ordering is absent. Thus, it can be concluded that at low $q$ the model evolves toward a low- $q$ state, or toward a set of low- $q$ states, characterized by the occurrence of the short-range ordering, which corresponds to the SG-like phase. Such state differs qualitatively from the disordered state observed at higher $q$. Moreover, analysis of the Binder cumulants $U^{(Q)}$ suggests that there is a critical value $q_{c}$ for the appearance of the SG-like phase. Hence, it can be argued that for a certain range of parameters SG-like transition can occur in the model under study; however, it should be remembered that evidence for this transition is purely phenomenological since it cannot be proved that the configuration of agents' opinions in the SG-like phase corresponds to any stable equilibrium.

\section{Results and discussion}

Let us first consider the MV model on complex networks in the case when $p=1$, i.e., when all agents are anticonformists. On the one hand, from a sociological point of 


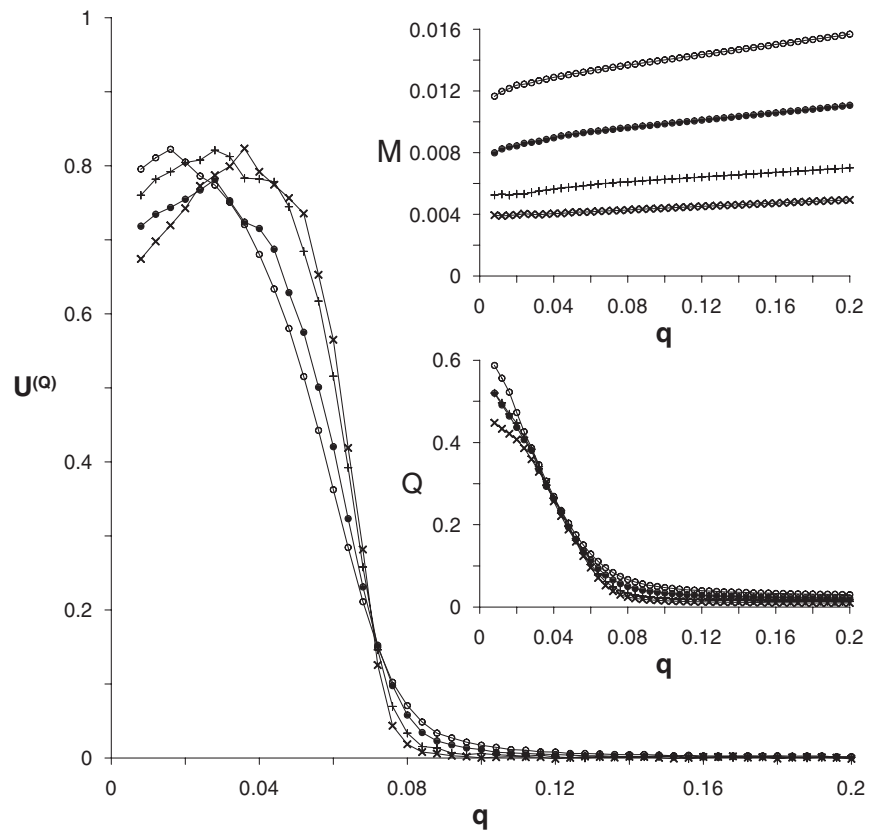

Fig. 1. Binder cumulants $U^{(Q)}$ vs. $q$ for the model with $p=1.0$ (all agents are anticonformists) on SF network with $m=10$, $\gamma=4.5$, and (o) $N=1000,(\bullet) N=2000,(+) N=5000,(\times)$ $N=10000$. Insets: magnetization $M$ vs. $q$ (top) and the SG order parameter $Q$ vs. $q$ (bottom).

view this case is not interesting since it is hard to imagine a society consisting of only anticonformists; on the other one, the combined effect of the disorder in network connections and of the local AFM opinion update rule, equation (4), can lead to the occurrence of local AFM ordering typical of the SG-like phase. In Figure 1 dependence of the order parameters $M, Q$ and of the Binder cumulant $U^{(Q)}$ on $q$ is shown for the model on SF network with $\gamma=4.5$; qualitatively similar results were obtained in the case of SF networks with $\gamma>3$ and random ER graphs. It can be seen that the SG order parameter $Q$ increases significantly from zero as $q$ is decreased for all network sizes $N$ while the magnetization $M$ remains close to zero or even decreases, which suggests the occurrence of the SG-like transition. The cumulants $U^{(Q)}$ are in general mononically decreasing functions of $q$ which suggests that the transition is second-order, and the curves for different $N$ intersect at one point $q_{c} \approx 0.07$ which is the critical value of the internal noise. The occurrence of the continuous transition to the SG-like phase is also confirmed in Figure 2 where it is shown that the distribution $P(Q)$ of the overlap parameter changes smoothly from unimodal in the disordered phase to bimodal in the SG-like phase. Further evidence for the appearance of the SG-like phase is provided by the behavior of the correlation function $C(r)$ (Fig. 3). For low $q$ the function $C(r)$ oscillates around zero and at $r=1$ shows a negative minimum which proves that nearest neighbors have, on average, opposite opinions, i.e., short-range AFM ordering appears in the model (Fig. 3a). Besides, at least for larger $r$ approximate exponential scaling $|C(r)| \propto \exp \left(-r / r_{0}\right)$ is observed, with the characteristic distance $r_{0}$ in the case of SF networks increasing

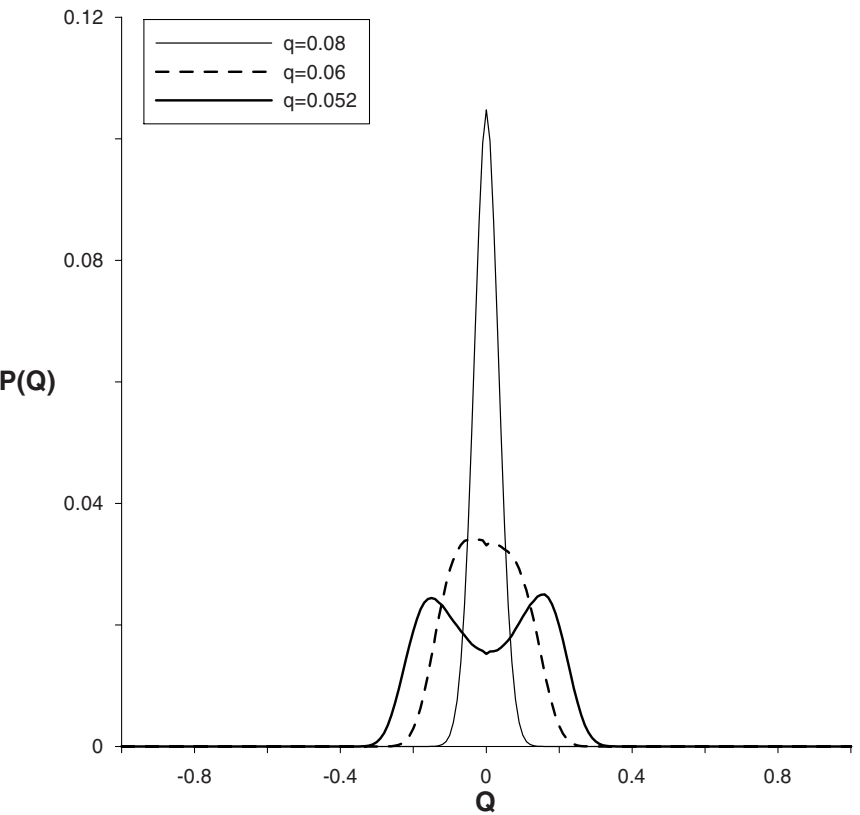

Fig. 2. Distributions of the SG order parameter $P(Q)$ for the model with $p=1.0$ (all agents are anticonformists) on SF network with $m=10, \gamma=4.5$, and $N=5000$ for different values of $q$ (see legend). In order to evaluate the distributions sets of $5 \cdot 10^{4}$ values of $Q$ were obtained by performing averages over short time intervals and small sets of different realizations of the network in equation (6).

with $\gamma$ and approaching that for the case of random ER graphs, i.e., long-range ordering is absent in the model (Fig. 3b).

The above-mentioned results show that the critical behavior of the MV model with purely anticonformist agents on complex networks qualitatively resembles that of the Ising model with purely AFM exchange interactions, where the SG transition was confirmed both theoretically, using the replica method [11,12], and by means of MC simulations [4,5]. However, it should be noted that though both the SG order parameter $|Q|$ and the Binder cumulant $U^{(Q)}$ significantly increase for $q \rightarrow 0$ they do not reach the limits $Q \rightarrow 1, U^{(Q)} \rightarrow 1$. This may be related to the fact that the low- $q$ state corresponding to the SG-like phase need not be unique or that reaching this state (or states) using the simulated annealing algorithm requires prohibitively long simulation times.

In Figure 4 dependence of the order parameter $Q$ and of the Binder cumulant $U^{(Q)}$ on $q$ is shown for the MV model with $p=1$ on SF network with $\gamma=3$. At a first glance, appearance of a negative minima of the curves $U^{(Q)}$ vs. $q$ could indicate occurrence of the first-order SGlike transition. However, though the SG order parameter $Q$ increases as $q$ is decreased the Binder cumulants for different system sizes $N$ do not tend to intersect at one point. Qualitatively similar results were obtained for the model with $p=1$ on SF networks with $2<\gamma<3$. This suggests that in the model under study on networks with divergent second moment of the degree distribution $\left\langle k^{2}\right\rangle$ the SG-like transition does not occur. This is in contrast 

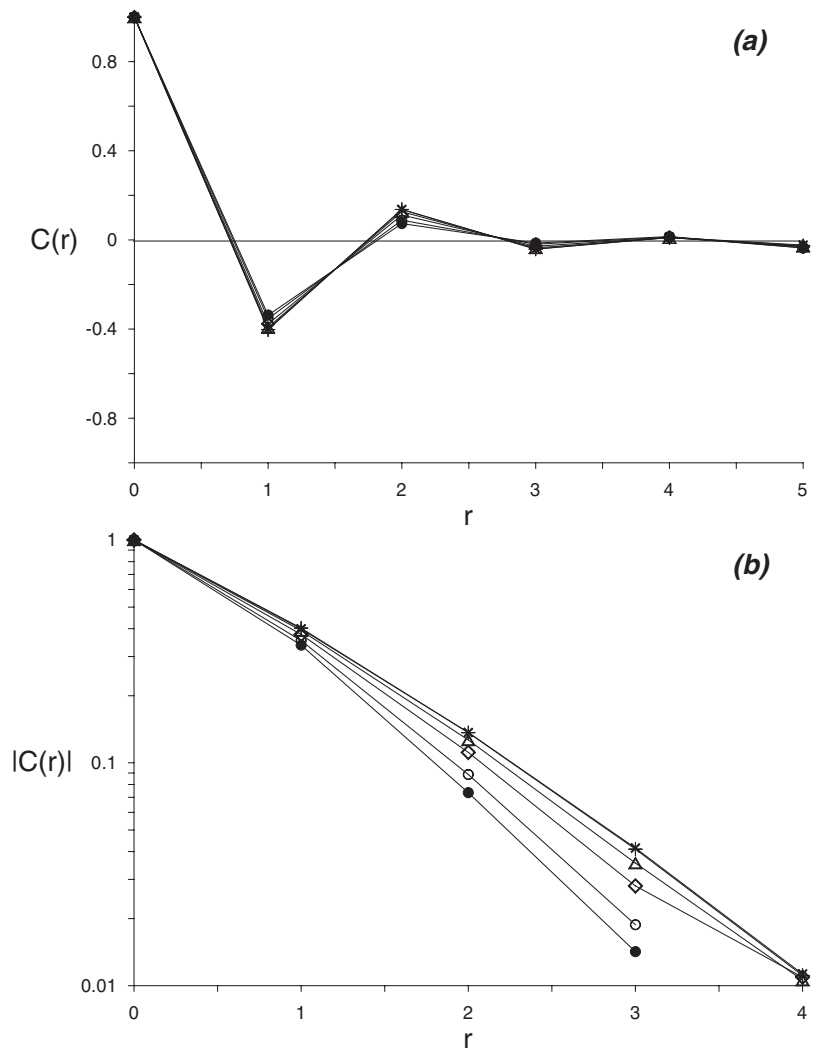

Fig. 3. (a) Correlation function $C$ and (b) absolute value of the correlation function $|C|$ vs. distance (shortest path) between nodes $r$ at $q=0.02 \ll q_{c}$ for the model with $p=1.0$ (all agents are anticonformists), $N=10000$ on SF networks with $m=10$ and $(\bullet) \gamma=3.25,(\circ) \gamma=3.5,(\diamond) \gamma=4,(\triangle) \gamma=4.5,(+)$ $\gamma=5$ and on $(\times)$ random ER graphs with $\langle k\rangle=13$.

with the critical behavior of the MV model with $p=0$ (in which all agents are conformists) on SF networks, investigated in reference [23], which shows FM transition for $\gamma>2.5$, i.e., even if $\left\langle k^{2}\right\rangle$ diverges, provided that $\left\langle k^{3 / 2}\right\rangle$ is finite (see Eq. (3)).

In the MV model on SF networks with $\gamma>3$ and on random ER graphs the SG-like transition occurs also for narrow intervals of $p \leq 1$, i.e., in societies with a huge majority of anticonformists; e.g., for SF network with $\gamma=4.5$ this interval is approximately $0.97<p \leq 1$ (Fig. 5). The critical value $q_{c}$ for the SG-like transition decreases fast with decreasing $p$. For small values of $p$, i.e., in societies in which most agents are usual conformists, the FM transition is observed, characterized by the increase of both order parameters $M, Q$ as $q$ is decreased, and by intersection of the Binder cumulants $U^{(M)}$ for different $N$ at one point $q_{c}$ (Fig. 6); e.g., for SF network with $\gamma=4.5$ this interval is approximately $0 \leq p \leq 0.3$. The critical value $q_{c}$ for the FM transition decreases with increasing $p$. The phase diagram for the MV model on SF network with $\gamma=4.5$ is shown in Figure 7, where PM denotes the disordered "paramagnetic" phase. It is interesting to note that this diagram differs qualitatively from that for the

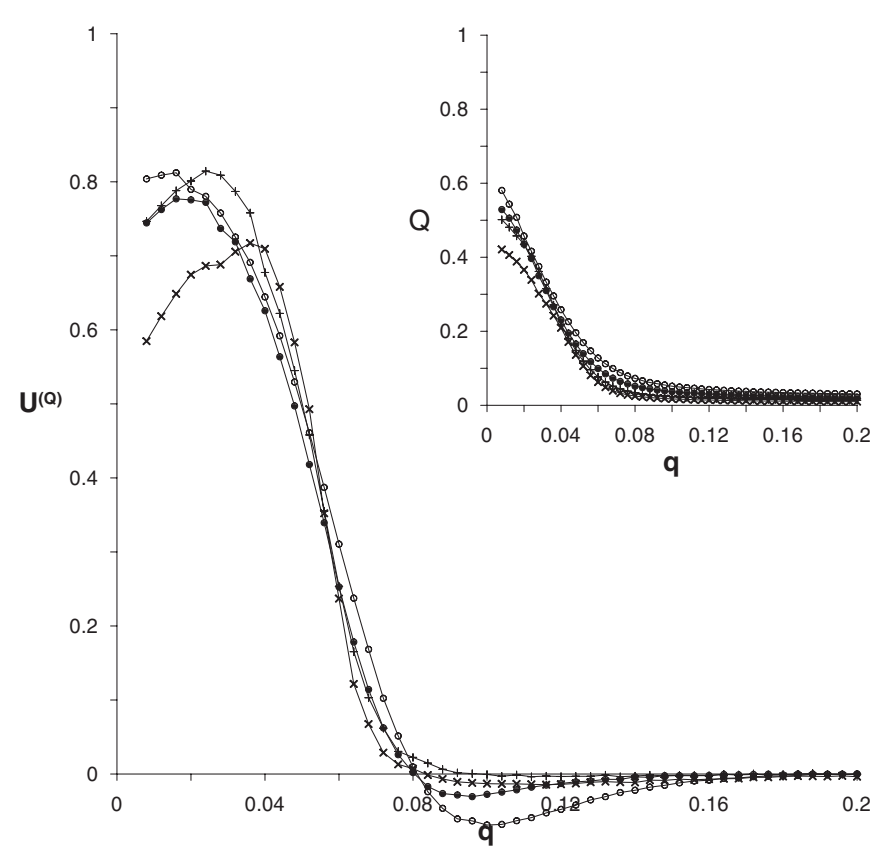

Fig. 4. Binder cumulants $U^{(Q)}$ vs. $q$ for the model with $p=1.0$ (all agents are anticonformists) on SF network with $m=10$, $\gamma=3.0$, and (o) $N=1000,(\cdot) N=2000,(+) N=5000,(\times)$ $N=10000$. Inset: SG order parameter $Q$ vs. $q$.

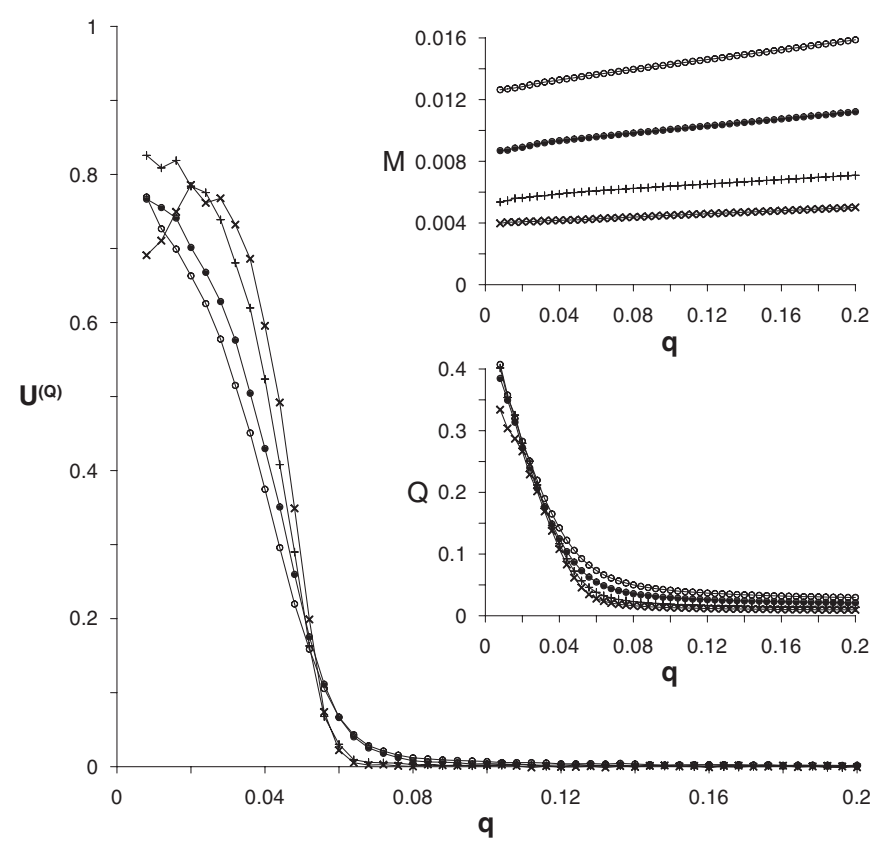

Fig. 5. Binder cumulants $U^{(Q)}$ vs. $q$ for the model with $p=$ 0.98 on SF network with $m=10, \gamma=4.5$, and (o) $N=1000$, $(\bullet) N=2000,(+) N=5000,(\times) N=10000$. Insets: magnetization $M$ vs. $q$ (top) and the SG order parameter $Q$ vs. $q$ (bottom). 


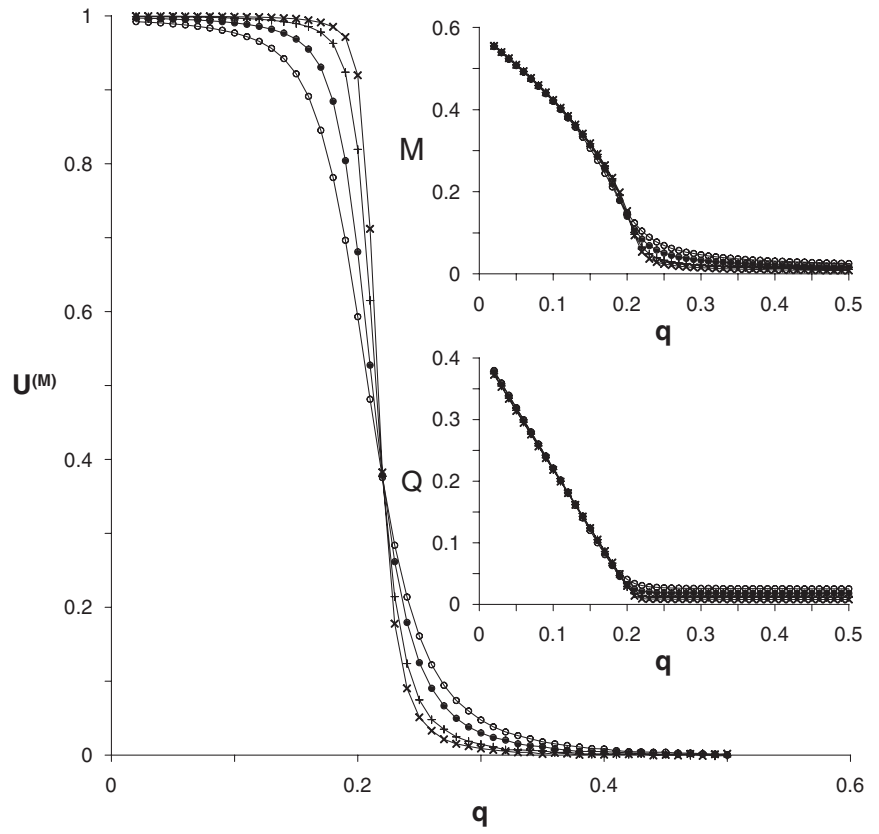

Fig. 6. Binder cumulants $U^{(M)}$ vs. $q$ for the model with $p=0.2$ on SF network with $m=10, \gamma=4.5$, and (o) $N=1000$, (•) $N=2000,(+) N=5000,(\times) N=10000$. Insets: magnetization $M$ vs. $q$ (top) and the SG order parameter $Q$ vs. $q$ (bottom).

Ising models for dilute spin glasses on random ER graphs and SF networks [11,12]: for intermediate values of $p$ only disordered phase is observed and no phase transition occurs for $q \rightarrow 0$, thus, in particular, there is no tricritical point at which the borders between the disordered and the FM phase as well as between the disordered and the SG phase merge. The problem if this difference can be attributed to the asymmetry of interactions between agents (see Sect. 2.1) or is a general property of the MV model requires further investigation. Another interesting difference occurs between the MV model with $p=1$ and the Ising model with purely AFM exchange interactions on SF networks: in the former case the critical value $q_{c}$ for the SG-like transition is only weakly dependent on the exponent $\gamma$ in the power scaling law for the degree distribution, in contrast with what was reported in the latter case [5].

\section{Conclusions}

The nonequilibrium MV model on complex networks in which a fraction of agents are anticonformists was investigated by means of MC simulations. In the case of networks with finite second moment of the degree distribution different phase transitions were observed as the level of internal noise was decreased: the FM transition if most of the agents were conformists and the SG-like transition if all or almost all agents were anticonformists. In the latter case it is not possible to provide theoretical arguments that the SG-like phase corresponds to any "ground" state. Nevertheless, phenomenological evidence

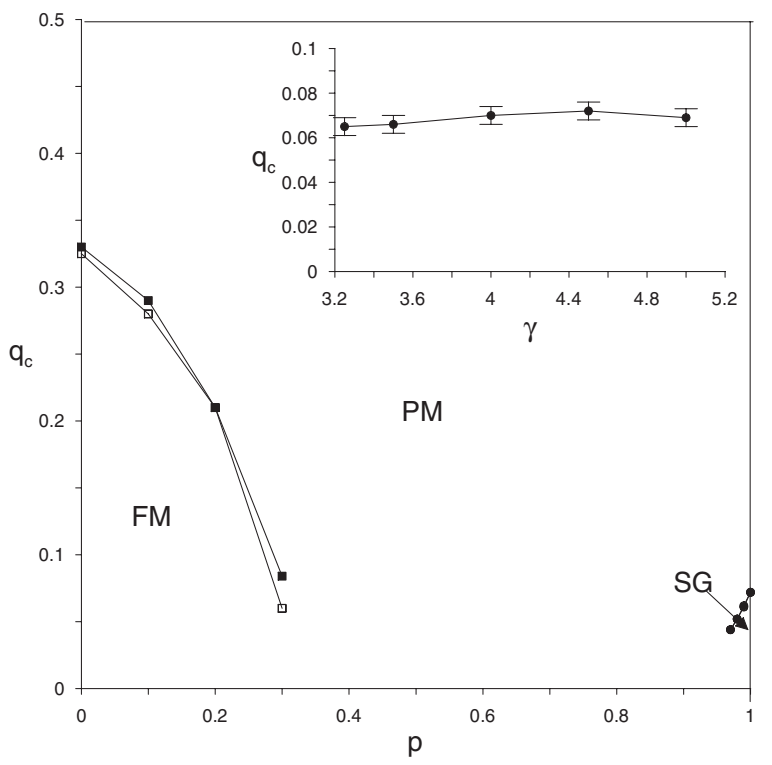

Fig. 7. Phase diagram for the MV model with anticonformists on complex networks. Open symbols correspond to the model on random ER graph with $\langle k\rangle=13$, filled symbols to the model on SF network with $m=10, \gamma=4.5$ (borders between the PM and SG phases coincide for both kinds of networks; phase borders from the intersection points of the respective Binder cumulants for different $N$ could be reliably determined only for $\left.q_{c} \geq 0.05\right)$. Inset: $q_{c}$ vs. $\gamma$ for the SG-like transition in the model with $p=1.0$ on SF networks with $m=10$.

based on MC simulations and the use of typical quantities (order parameters, Binder cumulants, correlations between agents' opinions) suggests that the SG-like transition occurs and that for low levels of the internal noise the model approaches a low- $q$ state (or a set of low- $q$ states) characterized by short-range ordering and lack of longrange ordering. Taking into account the above-mentioned results it would be interesting to check if in other nonequilibrium systems similar transitions to phases with only short-range ordering are possible.

\section{Author contribution statement}

The author (A.K.) is responsible for the whole content of the paper.

Open Access This is an open access article distributed under the terms of the Creative Commons Attribution License (http://creativecommons.org/licenses/by/4.0), which permits unrestricted use, distribution, and reproduction in any medium, provided the original work is properly cited.

\section{References}

1. S.N. Dorogovtsev, A.V. Goltsev, J.F.F. Mendes, Rev. Mod. Phys. 80, 1275 (2008)

2. A. Aleksiejuk, J.A. Hołyst, D. Stauffer, Physica A 310, $260(2002)$ 
3. C.P. Herrero, Phys. Rev. E 69, 067109 (2004)

4. M. Bartolozzi, T. Surungan, D.B. Leinweber, A.G. Williams, Phys. Rev. B 73, 224419 (2006)

5. C.P. Herrero, Eur. Phys. J. B 70, 435 (2009)

6. C.P. Herrero, Phys. Rev. E 91, 052812 (2015)

7. G. Bianconi, Phys. Lett. A 303, 166 (2002)

8. S.N. Dorogovtsev, A.V. Goltsev, J.F.F. Mendes, Phys. Rev. E 66, 016104 (2002)

9. S. Yoon, A.V. Goltsev, S.N. Dorogovtsev, J.F.F. Mendes, Phys. Rev. E 84, 041144 (2011)

10. M. Leone, A. Vázquez, A. Vespignani, R. Zecchina, Eur. Phys. J. B 28, 191 (2002)

11. L. Viana, A.J. Bray, J. Phys. C: Solid State Phys. 18, 3037 (1985)

12. D.-H. Kim, G.J. Rodgers, B. Kahng, D. Kim, Phys. Rev. E 71, 056115 (2005)

13. D.-H. Kim, Phys. Rev. E 89, 022803 (2014)

14. A. Krawiecki, Physica A 492, 534 (2018)

15. M.J. Oliveira, J. Stat. Phys. 66, 273 (1992)

16. M.J. Oliveira, J.F.F. Mendes, M.A. Santos, J. Phys. A: Math. Gen. 26, 2317 (1993)

17. J.-S. Yang, I.-M. Kim, W. Kwak, Phys. Rev. E 77, 051122 (2008)

18. A.L. Acuña-Lara, F. Sastre, Phys. Rev. E 86, 041123 (2012)

19. L.F.C. Pereira, F.G. Brady Moreira, Phys. Rev. E 71, 016123 (2005)

20. P.R.A. Campos, V.M. de Oliveira, F.G. Brady Moreira, Phys. Rev. E 67, 026104 (2003)
21. F.W.S. Lima, Int. J. Mod. Phys. C 17, 1257 (2006)

22. F.W.S. Lima, Commun. Comput. Phys. 2, 358 (2007)

23. H. Chen, C. Shen, G. He, H. Zhang, Z. Hou, Phys. Rev. E 91, $022816(2015)$

24. F. Sastre, M. Henkel, Physica A 444, 897 (2016)

25. A.L.M. Vilela, F.G. Brady Moreira, Physica A 388, 4171 (2009)

26. A.R. Vieira, N. Crokidakis, Physica A 450, 30 (2016)

27. A.S. Balankina, M.A. Martínez-Cruza, F. Gayosso Martínez, B. Mena, A. Tobon, J. Patiño-Ortiz, M. PatiñoOrtiz, D. Samayoa, Phys. Lett. A 381, 440 (2017)

28. H. Chen, C. Shen, H. Zhang, G. Li, Z. Hou, J. Kurths, Phys. Rev. E 95, 042304 (2017)

29. A.-L. Barabási, R. Albert, Science 286, 509 (1999)

30. R. Albert, A.-L. Barabási, Rev. Mod. Phys. 74, 47 (2002)

31. P. Erdös, A. Rényi, Publ. Math. 6, 290 (1959)

32. K. Binder, A.P. Young, Rev. Mod. Phys. 58, 801 (1986)

33. M. Mézard, G. Parisi, M.A. Virasoro, Spin glass theory and beyond (World Scientific, Singapore, 1987)

34. H. Nishimori, Statistical physics of spin glasses and information theory (Clarendon Press, Oxford, 2001)

35. K. Binder, D. Heermann, Monte Carlo simulation in statistical physics (Springer-Verlag, Berlin, 1997)

36. K. Hukushima, K. Nemoto, J. Phys. Soc. Jpn. 65, 1504 (1996)

37. H.G. Katzgraber, M. Palassini, A.P. Young, Phys. Rev. B 63, $184422(2001)$

38. H.G. Katzgraber, A.P. Young, Phys. Rev. B 65, 214402 (2002) 